\title{
A Study of COVID-19 Vaccine (COVISHIELD) Pharmacovigilance in Primary Healthcare Workers in Punjab, India
}

\author{
Rahat Kumar ${ }^{1}$, Jaswinder Singh ${ }^{2}$, Narinder Singh ${ }^{3}$, Vikram Bhandari ${ }^{4}$
}

\begin{abstract}
Aims and Objectives: Coronavirus disease-2019 (COVID-19) is caused by a novel RNA coronavirus that can cause lethal diseases, such as severe acute respiratory syndrome (SARS) and Middle East respiratory syndrome. Symptom onset is from 3 to 5 days with mild- to high-grade fever, persistent cough, mild to severe dyspnea with fall in $\mathrm{SpO}_{2}$, anosmia, ageusia, and fatigue. The management of COVID-19 infection includes oxygen, antiviral drugs like lopinavir or ritonavir, remdesivir, oseltamivir, favipiravir, ventilatory support, antibiotics, etc. Unfortunately, $100 \%$ recovery rate is not seen, and many patients die due to complications, so the main emphasis is on vaccine administration for the induction of virus-neutralizing antibodies. This study was done to assess the safety of Covishield in primary healthcare workers so as to assess the risk assessment in general population.

Materials and methods: Covishield (ChAdOx1 nCoV-19), a coronavirus vaccine (recombinant), is manufactured by the Serum Institute of India Pvt. Ltd. Pune, India. Covishield has been approved for emergency authorization against COVID-19 infection. Covishield during phase I vaccination drive after consent was given to the 1,054 healthcare workers of Sri Guru Ram Das Institute of Medical Sciences and Research (SGRDIMSAR), Amritsar, and safety data were generated in postvaccination phase.

Results: It was observed that there were $97.3 \%$ of solicited reactions and $2.7 \%$ of unsolicited reactions. Most of the solicited reactions were pain at the injection site, mild fever, malaise, and headache with a duration of symptoms generally for 2-3 days. In the unsolicited reactions, mainly moderate- to high-grade fever followed by decreased appetite and dizziness with a duration of 2-3 days were observed. Only in one subject, an increase in liver function test occurred for 3 days.

Conclusion: The findings of our study indicate that Covishield vaccine has provided excellent tolerability in health care subjects who willingly received the vaccine during phase I mass vaccination, and there was no report of any untoward side effects demanding attention in such patients. Clinical significance: The tolerability of Covishield vaccine observed in the present study can be used to extrapolate the safety in the general population. Thus, mass vaccination with Covishield is recommended in the general population without any fear of vaccine-related serious side effects. Keywords: Coronavirus, Covishield, Pharmacovigilance, SARS-CoV-2.

AMEl's Current Trends in Diagnosis \& Treatment (2021): 10.5005/jp-journals-10055-0111
\end{abstract}

\section{INTRODUCTION}

Coronavirus disease-2019 (COVID-19) is caused by novel severe acute respiratory syndrome coronavirus 2 (SARS-CoV-2), which is a group of RNA viruses that cause mild to lethal disease in humans, that is, common cold to lethal infections, such as SARS and Middle East respiratory syndrome. The onset of symptoms is generally around 3-5 days from the start of initial infection, and the most frequently experienced symptoms are mild- to high-grade fever, persistent cough, mild to severe dyspnea with fall in $\mathrm{SpO}_{2}$, anosmia, ageusia, and fatigue. The first identified outbreak of COVID-19-induced respiratory illness was reported in Wuhan City, Hubei Province, China. ${ }^{1}$ The terminology of COVID-19 was given by the World Health Organization (WHO) as the term was taken from "coronavirus disease 2019." The purpose of naming the disease as Corona was to prevent stigmatizing the virus in terms of origins in any specific populations, geographical location, or animal associations. ${ }^{2}$ As per the Center for Disease Control (CDC), the individuals who are prone to have higher risk of infection are the persons having comorbidities, contact with local transmission, travelers returning from the countries or areas having higher prevalence or spread of infection, healthcare workers associated with caring of COVID-19 patients like critical acre specialists, anesthetists, physicians, and close family

\footnotetext{
${ }^{1-4}$ Department of Pharmacology, SGRDIMSAR, Amritsar, Punjab, India

Corresponding Author: Rahat Kumar, Department of Pharmacology, SGRDIMSAR, Amritsar, Punjab, India, Phone: +91 9876125136, e-mail: rahat_sharma66@yahoo.com

How to cite this article: Kumar R, Singh J, Singh N, et al. A Study of COVID-19 Vaccine (COVISHIELD) Pharmacovigilance in Primary Healthcare Workers in Punjab, India. AMEl's Curr Trends Diagn Treat 2021;5(1):6-11.

Source of support: Nil

Conflict of interest: None
}

members, or contacts with infected patients. The presentation of COVID-19 infection as asymptomatic, mild symptoms, or severe critical illness with multisystemic involvement may lead to recovery or death. Classically, the duration of symptoms ranges from 2 days to 2 weeks after the exposure to the infected patients. ${ }^{3}$ As of February 8, 2021, over 105 million confirmed COVID-19 infections globally have been reported with over 2.3 million deaths. ${ }^{4}$ The origin of the COVID-19 pandemic was from Wuhan, China, to all over the world by person-to-person transmission, making it the worst pandemic after the Spanish flu that occurred in $1918 .^{5}$ COVID-19 
has affected all the sections of the society globally, from rich to poor, and has resulted in an unexpected rise in health care cost, economic loss in families due to loss of job, and mental trauma due to sudden unexpected death occurring in family members. The COVID-19 pandemic in India and worldwide is one of the worst of its kind of communicable infection in the human species. The WHO Country Office, India, and Government of India (GOI) have been working collaboratively since the onset of the COVID-19 pandemic for quick responsive measures, such as surveillance, contact tracing, protective measures, such as use of mask and sanitizers, hightech laboratory sampling (i.e., reverse transcription-polymerase chain reaction and rapid antigenic testing), and educating and communicating to public by community engagement and participation. The hospitals have been directed for their emergency preparedness for diagnosis, treatment with ventilator provision, and implementation of containment plan at all three levels of the healthcare system: national, state, and district levels. As of February 2021, a total number of cases reported have been 137,342 with deaths of 156,014 and the number of discharged or migrated being $10,656,845 / 1 .^{6}$ The management of COVID-19 infection includes antiviral drugs like lopinavir or ritonavir, remdesivir, oseltamivir, and favipiravir, but there is no $100 \%$ of the efficacy of these agents in acute infectious episodes with high mortality. Treatment of COVID-19 infection is mainly symptomatic with oxygen therapy and management of multiple complications including the most important being severe respiratory distress to prevent mortality in such patients. The invasive and invasive mechanical ventilation may be required in cases of respiratory failure refractory to oxygen therapy. Prevention is the best method for the prevention of virus propagation and is achieved by regular handwashing using soap or sanitizers, avoiding touching of the nose and face, and adopting $6 \mathrm{ft}$ social distancing from each other. Because there is no specific drug for COVID-19 infection, the main emphasis is on vaccination. In India, the world's largest Covid mass vaccination drive started on January 16 , and after the start of the vaccination, within 18 days, the country has successfully vaccinated over 4.1 million healthcare workers. ${ }^{7}$ Worldwide pharmaceutical companies and governments have developed various vaccines using their patent technologies, but the aim of the vaccines for COVID-19 infection is to induce long-term persistent antibody formation that can neutralize SARS-CoV-2 virus and hence the complications. The vaccines after being administered in the body induce antibody formation by stimulating cellular immune responses. The rapid surge in these antibodies can limit the replication of the virus within the infected host by the opsonization of these viruses. The main emphasis of vaccine formulation is on the induction of virus-neutralizing antibodies directed against the SARS-CoV-2 spike (S) protein after the vaccine is injected. ${ }^{8}$ The unfolded protein response induction is mediated mainly by viral spike $(S)$ protein. $S$ protein is made-up of two subunits: S1 subunit having a receptor-binding domain on the angiotensin-converting enzyme 2 receptor named as AT-2 and another subunit S2 that mediates fusion between the viral and host cell membranes. ${ }^{9} \mathrm{~S}$ protein is important in the induction of neutralizing antibody and protective immunity, and T-cell humoral responses in patients infected with COVID-19 infection. The most commonly used worldwide vaccines are as follows: ${ }^{10}$

1. Oxford University and AstraZeneca

2. CanSino Biological Incs. and Beijing Institute of Biotechnology.

3. Moderna and National Institute of Allergy and Infectious Diseases
1. Oxford University and AstraZeneca: Oxford University is a UK-based university working in collaboration with UK-Swedish drug company AstraZeneca and has prepared ChAdOx1 nCoV-19 vaccine against COVID-19. The ChAdOx1 $\mathrm{nCoV}-19$ vaccine is formulated using the replication-deficient

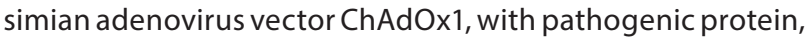
namely $S$ protein, of SARS-CoV-2, which is the most common strain for invading pathogens in human cells. ${ }^{11}$ This vaccine (ChAdOx1 nCoV-19) was developed at Oxford University and consists of a replication-deficient chimpanzee adenoviral vector ChAdOx1, containing the SARS-CoV-2 structural surface glycoprotein antigen (spike protein; nCoV-19) gene. Covishield is a trademark of ChAdOx $1 \mathrm{nCoV}-19$, and the coronavirus vaccine (recombinant) manufactured by this technology is marketed by the Serum Institute of India Pvt. Ltd., 212/2, Hadapsar, Pune 411 028, India. ${ }^{12}$

2. Ad5-nCOV-COVID-19 vaccine: It is manufactured by a Chinese company CanSino Biological Incs. in collaboration with Beijing Institute of Biotechnology. Ad5-nCOV-COVID-19 vaccine-is made in collaboration with Beijing Institute of Biotechnology, and this vaccine has shown good results in phase III clinical trials. Nonreplicating, noninfectious adenovirus 5 (Ad5) vector carrying the gene coding for SARS-CoV-2 spike protein $S$ was manufactured and then was injected intramuscularly in healthy human volunteers aged between 18 and 60 years, and the vaccination generated antibodies with the adequate antibody titer signifying the immunological response of the vaccine. The Ad5-nCOV-COVID-19 vaccine-was shown to be safe and effective with maximum humoral (antibody formation) responses against SARS-CoV-2 seen after 28 days of vaccination, and the rapid specific T-cell-mediated responses were seen at 14 days postvaccination. ${ }^{13}$

3. Moderna: It is manufactured in collaboration with the National Institute of Allergy and Infectious Diseases. Moderna is a US-based mRNA-1273 vaccine and has shown good results in phase 2 and 3 clinical trials with an efficacy of around $94.1 \%$. Moderna, a mRNA-1273-based vaccine, is formulated after targeting the SARS-CoV-2 spike (S) protein. The manufacturing procedure was selected in collaboration with investigators from the Vaccine Research Center at the National Institute of Allergy and Infectious Diseases, which is a part of the National Institute of Health. ${ }^{14}$ Moderna is currently given to the healthcare workers and high-risk subjects in the USA. The vaccine has proved to be efficacious and safe with only minor tolerable side effects.

\section{India's Coronavirus Vaccine}

The pace of the spread of coronavirus is different worldwide, and in fact, some countries are facing a second wave of attack. So, pharmaceutical companies have started preparing the COVID-19 vaccine, and even some have got success in this area. Various laboratories in India had been trying to develop a vaccine against COVID-19 like Serum Institute of India, Bharat Biotec, Panacea Biotec, and Zydus Cadila. On January 1, 2021, the Drug Controller General of India, approved the emergency or conditional use of AstraZeneca's COVID-19 vaccine (branded as Covishield) in the people of India. Similarly, Bharat Biotech's "COVAXIN" ${ }^{\mathrm{TM} \text { " has }}$ received emergency use. ChAdOx1 nCoV-19 vaccine (Covishield) was developed at Oxford University, and this vaccine is formulated using a nonreplicating chimpanzee adenoviral vector ChAdOx1, 
known to cause cold in chimpanzees. ${ }^{15}$ This vector has a structural surface glycoprotein antigen (spike protein; nCoV-19) gene similar to SARS-CoV-2, and thus, this vaccine is a viral vector vaccine based on replication-deficient. The good point about Covishield vaccine is that it can be stored, transported, and handled at normal refrigerator conditions $\left(2-8^{\circ} \mathrm{C}\right)$ and has got a shelf life of at least 6 months. As per the recommendation, the vaccine dosage is given in two doses from 4 to 12 weeks apart. The duration of protection by this schedule is still in the infancy stage as it is not possible to estimate the long-term protection at this juncture. India is currently one of the leading manufacturers and suppliers of Covishield vaccines in the world, and it accounts for around $60 \%$ of the total vaccines supplied to UNICEF. The cost of manufacturing and clinical trials in India is relatively lower than that in developed countries, so there is a huge scope for this vaccine supply and distribution worldwide with resultant huge profit for India. ${ }^{16}$

\section{Clinical Efficacy Data of COVISHIELD Vaccine}

The efficacy and safety of ChAdOx1 nCoV-19 (Covishield) vaccine were evaluated in four randomized, controlled trials conducted in the diverse population of the United Kingdom, South Africa, and Brazil. In the pooled data report of chimpanzee adenovirus vectored vaccine ChAdOx1 $\mathrm{nCoV}-19$ (Covishield) vaccine given to 5,807 subjects of age 18 years and above, it was seen that the efficacy of COVID-19 (ChAdOx1 nCoV-19) vaccination after 14 days of the second dose was $70.4 \%$ versus other group in the control group showing that Covishield vaccine was safe and effective with no serious adverse events (SAEs) or deaths reported. ${ }^{17}$

\section{Vaccine Pharmacovigilance Program in India against COVID-19 Infection}

Vaccine pharmacovigilance is defined as the science and activities relating to the detection, assessment, understanding, prevention, and communication of adverse events following immunization (AEFI), or of any other vaccine-or immunization-related issues. ${ }^{18}$ Currently, under mass vaccination campaign in India, both Covishield and Covaxin are given to healthy subjects who have willing to be enrolled for vaccination. In India, the world's biggest COVID-19 vaccination programs against COVID-19 infection has started, thereby embarking on the beginning of an effort to immunize more than 1.3 billion people. However, the data showing the efficacy and safety of corona vaccination are not available at present, so this article was planned to focus on the safety of the COVID-19 vaccination program by estimating the adverse events (AEs)/ and pharmacovigilance following vaccination in subjects given Covishield injection and to establish any association of AEs with associated illness.

\section{Goal of COVISHIELD Vaccine Pharmacovigilance}

Early detection and timely management of AEs in order to minimize morbidity or mortality in subjects following immunization with Covishield in population.

\section{Need of Vaccine Pharmacovigilance}

COVID-19 pandemic has led to an emergency authorization for the use of COVID-19 vaccination in various countries, including India. Clinical trials of a vaccine often involve a limited number of participants but exclude immunocompromised patients, pregnant women, children, and patients with comorbid conditions like diabetes, cardiac problems, immunological diseases, taking corticosteroids, etc. Thus, data are needed to establish the safety of Covishield vaccine in subjects given vaccine so as to establish the benefits-risk ratio in population.

\section{Materials and Methods}

This was an observational, retrospective, noninterventional, passive surveillance safety study with no formal sample size. The study was based on spontaneous report following Covishield vaccination in healthcare workers. The Covishield vaccination was given by the Immunization Department, PSM (Preventive and Social Medicine) Department, Sri Guru Ram Das Hospital, a charitable hospital attached to the medical institute Sri Guru Ram Das Institute of Medical Sciences and Research (SGRDIMSAR), Amritsar, Punjab, India. The subjects willing for Covishield vaccination were given the vaccine, and following vaccination, subjects were observed for half an hour for any untoward event. The subjects after the consent were given a single dose of $0.5 \mathrm{~mL}$ of Covishield vaccine intramuscularly. The immunocompromised subjects like taking any immunosuppressive drug were excluded to give the vaccination. Subjects were instructed to record any solicited local and systemic reactions within 7 days and all potential unsolicited events within 42 days postvaccination. A diary card was given to improve the reporting, and subjects were advised to report any $A E$ on WhatsApp group that has been created by the author. Afterward, the subjects were encouraged to report solicited and unsolicited AEs within 7 days from vaccination by personal interaction, telephonic reporting, and even by reporting via WhatsApp at pharmacovigilance cell, SGRDIMSAR, Amritsar, Punjab, India. The investigator reviewed and transcribed the information in case report forms (CRFs) for the analysis. The Covishield vaccine was provided by the GOI under the phase I vaccination program for the frontline healthcare workers. The vaccine was given at left arm, that is, left deltoid region after sterilization of the area and following the standard operating procedure of vaccine administration program. The dosage of Covishield ${ }^{\mathrm{TM}}$ vaccination course consists of two separate doses of $0.5 \mathrm{~mL}$ each. The second dose should be administered between 4 and 8 weeks after the first dose. The solicited and unsolicited reactions after vaccination till 7 days were noted on the proforma designed as per the literature provided by the Serum Institute of India using the following parameters: tenderness (>60\%); injection site pain, headache, and fatigue ( $>50 \%)$; myalgia and malaise ( $>40 \%)$; pyrexia and chills (>30\%); and arthralgia and nausea (>20\%). ${ }^{19}$ The data collected from the subjects given Covishield vaccine were further analyzed for their appropriateness and suitability. The unsolicited adverse drug reactions (ADRs) were reported to the central pharmacovigilance center at PvPI, CDSCO, IPC, Ghaziabad, India, under the pharmacovigilance program of India. The investigator reviewed and transcribed the information in CRFs.

\section{Study Objectives}

The study was planned to document solicited and unsolicited AEs following immunization and hence to establish the safety of Covishield vaccination.

\section{Rescue Medicine}

Paracetamol $650 \mathrm{mg}$ SOS, max up to four times a day as an analgesic agent, and domperidone $10 \mathrm{mg}$ SOS, max $10 \mathrm{mg}$ twice a day for nausea or vomiting, was given as a rescue drug. Severe reactions were managed according to the standard treatment guidelines of the diseases. 


\section{Statistical Methods}

All data were anonymized, and analyses were performed on all the subjects who participated in the study. It was an observational, noninterventional study where vaccination with the consent of the participants was given. The number of the subjects experiencing an $A E, S A E$, or $A D R$, and the number experiencing solicited or unsolicited events in each category were analyzed. The quantitative data were expressed in percentage for evaluation to show the relationships and comparisons between the category of responses.

\section{Ethical Approval}

This manuscript is the consent experience shared by the authors of this paper and is an observational, noninterventional study done to report the percentage of side effects of Covishield vaccine in subjects who willingly participated in the mass vaccination program.

\section{Results}

A total of 1,054 subjects were given Covishield vaccine in a dosage of $0.5 \mathrm{~mL}$ intramuscularly. The age distribution of the subjects given Covishield vaccination is given in Table 1. Out of 1,054 subjects, 664 (62.9\%) were males and 390 (37.1\%) were females, showing a higher vaccination for males than females in the mass vaccination program. It can also be interpreted from Table 1 that the most common age-group who participated in the mass vaccination program was from 40 to 50 years in 386 $(36.6 \%)$, while the subjects in the age-group $>50$ years enrolled were only 98 (9.2\%). Overall, there was a higher incidence rate of AEs observed in males as compared to females, 664 versus 390 (M:F = 1:70). Only three subjects were having comorbidities:

Table 1: Age distribution grouped by gender $(n=1,054)$

\begin{tabular}{llllc}
\hline Parameter & Age in years & Males & Females & Total (\%age) \\
\hline & $20-30$ & 185 & 96 & $281(26.6 \%)$ \\
& $30-40$ & 188 & 101 & $289(27.4 \%)$ \\
& $40-50$ & 217 & 169 & $386(36.6 \%)$ \\
& $>50$ & 74 & 24 & $98(9.2 \%)$ \\
Total & & $664(62.9 \%)$ & $390(37.1 \%)$ & $1,054(100 \%)$ \\
\hline
\end{tabular}

one with type 2 diabetes mellitus, one with rheumatoid arthritis, and one with coronary artery disease. The total number of adverse drug events (solicited and unsolicited observed) reported in subjects were 1,122, and out of the total AEs, 1,091 (97.3\%) were solicited and 31 (2.7\%) were unsolicited AEs. Out of all solicited 1,091 AEs reported in this retrospective study, the most common AEs were pain at the injection site (53.3\%), followed by a mild fever of temperature less than $100.5^{\circ} \mathrm{F}$ malaise (15\%), injection site swelling $(3.8 \%)$, headache $(4.4 \%)$, myalgia $(2.1 \%)$, chills $(1.3 \%)$, rigors $(1 \%)$, nausea $(1 \%)$, and nonsignificant events with less than one percentage was seen, such as arthralgia, influenza-like illness, and vomiting (Table 2). The mean duration of pain and mild fever of temperature less than $100.5^{\circ} \mathrm{F}$ was 2 days, myalgia and arthralgia were 3 days, but malaise, injection site swelling, headache, chills, rigors, nausea, influenza-like illness, and vomiting were seen 1 day. The AEs resolved spontaneously or were given symptomatic treatment for 1 to 2 days leaving no sequelae. Similarly, out of total 31 unsolicited AEs reported in this retrospective study, the most common AEs were $54 \%$ moderate- to high-grade fever with temperature greater than $100.6^{\circ} \mathrm{F}$ for 2 days, $22 \%$ decreased appetite for 2 days, and $16.1 \%$ dizziness for 2 days, while a nonsignificant $(<1 \%)$ AEs were pruritis for 1 day and increased liver function (slightly raised ALT/APT) for 3 days. However, there was no unsolicited event of generalized body rash, abdominal pain, hyperhidrosis, injection site hematoma, increased kidney function test, neurological problems, and lymphadenopathy (Table 3). All unsolicited AEs resolved with or without symptomatic treatment and without any sequelae. There was no SAE leading to deaths, prolonged hospitalization, permanent disability, events requiring hospitalization, or events requiring any visit to an emergency room. Similarly, there were no new-onset chronic medical conditions like optic neuritis and Guillain-Barré syndrome that were reported within 42 days of vaccine administration.

\section{Discussion}

The results of our study indicate that the Covishield (ChAdOx1 $\mathrm{nCoV}$-19) vaccine was safe and well tolerated in the healthcare workers who volunteered for vaccination. Since there is a raging

Table 2: Total number of solicited ADRs ( $n=1,091,97.3 \%)$

\begin{tabular}{llll}
\hline Adverse event & Number of subjects & $\%$ age & Mean duration (days) \\
\hline Pain at injection site & 584 & 53.5 & 2 \\
Mild fever (temperature $\leq 100.5^{\circ} \mathrm{F}$ ) & 201 & 18.4 & 2 \\
Malaise & 164 & 15 & 1 \\
Injection site swelling & 42 & 3.8 & 1 \\
Headache & 38 & 3.4 & 1 \\
Myalgia & 23 & 2.1 & 3 \\
Chills & 15 & 1.3 & 1 \\
Rigor & 12 & 1 & 1 \\
Nausea & 11 & 1 & 1 \\
Arthralgia & 04 & NS & 3 \\
Influenza-like illness & 03 & NS & 1 \\
Vomiting & 01 & NS & 1 \\
\hline
\end{tabular}

NS $=\%$ age $<1$ or zero value 
COVID-19 Vaccine (COVISHIELD) Pharmacovigilance in Primary Healthcare

Table 3: Total number of unsolicited ADRs ( $n=31,2.7 \%)$

\begin{tabular}{llll}
\hline Adverse event & Number of subjects & \%age & Mean duration in days \\
\hline Moderate- to high-grade fever (temperature $\left.\geq 100.6^{\circ} \mathrm{F}\right)$ & 17 & 54 & 2 \\
Decreased appetite & 07 & 22 & 2 \\
Dizziness & 05 & 16.1 & 2 \\
Pruritis & 01 & 0.03 & 1 \\
Increased liver function test & 01 & $\mathrm{NS}$ & 3 \\
Body rash & $\mathrm{Nil}$ & $\mathrm{NS}$ & $\mathrm{NS}$ \\
Abdominal pain & $\mathrm{Nil}$ & $\mathrm{NS}$ & $\mathrm{NS}$ \\
Hyperhidrosis & $\mathrm{Nil}$ & $\mathrm{NS}$ & $\mathrm{NS}$ \\
Injection site hematoma & $\mathrm{Nil}$ & $\mathrm{NS}$ & $\mathrm{NS}$ \\
Increased kidney function test & $\mathrm{Nil}$ & $\mathrm{NS}$ & $\mathrm{NS}$ \\
Neurological problem & $\mathrm{Nil}$ & $\mathrm{NS}$ & $\mathrm{NS}$ \\
Lymphadenopathy & $\mathrm{Nil}$ & $\mathrm{NS}$ & $\mathrm{NS}$ \\
\hline
\end{tabular}

NS $=\%$ age $<1$ or zero value

pandemic of COVID-19 infection throughout the world, safety is a major concern for the mass vaccination drive of subjects enrolling for vaccination. While the data presented here show that Covishield vaccine (ChAdOx1 $\mathrm{nCov}-19$ ) is safe in subjects with most AEs occurring in adults younger than 55 years of age. The findings of our study are in agreement with a study conducted by Voysey et al. ${ }^{20}$ who have shown that the subject participation was mostly of younger age-group for vaccination, but more of the females participated in their study for vaccination, which is in contrast to our findings where more of the male participation was seen for the voluntary vaccination program. Among 1,054 vaccinees, there was no report of a single severe or serious reaction endangering to life. Most of the AE reports were of solicited type (97.3\%). The most common $A E$ reported in our study was pain at the injection site (53.5\%), followed by mild fever (18.4\%) and malaise (15\%); and in less than $5 \%$ were injection site swelling, headache, myalgia, nausea, chills, and rigors; and in \%age less than one was seen arthralgia, vomiting, and influenza-like illness (Table 2). The mean duration of symptoms was 2 days for pain at injection site and mild fever, 3 days for arthralgia and myalgia, and 1 day for other symptoms. Similarly, $2.7 \%$ of unsolicited adverse reactions were observed in our study (Table 3). However, none of the participants included in this report had any serious adverse reactions threatening life. The most common AEs reported in unsolicited ADRs were $54 \%$ moderate- to high-grade fever (temperature $>100.6^{\circ} \mathrm{F}$ ) with a mean duration of 2 days, $22 \%$ decreased appetite with a mean duration of 2 days, and $16.1 \%$ dizziness with a mean duration of 2 days; one patient had pruritus that remained for 1 day only, and one patient (3\%) had slightly increased liver function test (LFT) that remained for 3 days only. However, there was no report of the generalized body rash, abdominal pain, hyperhidrosis, raised kidney function test, or lymphadenopathy (Table 3).

This is in line with AEs reported by Folegatti et al. and Tapia et al. ${ }^{21}$ phase 1 study of the ChAdOx 1 nCoV-19 vaccine where the results of the study indicate that the local and systemic reactions were commonly seen in participants of the Covishield vaccine (ChAdOx1 $\mathrm{nCoV}-19)$ than the subjects given control vaccine. The nature of AEs was also similar to those previously reported like pain at the injection site, feverish feeling, body aches, muscle ache, and headache. The mild tolerable AEs are positively correlated during COVID-19 vaccination as it is presumed that the body takes some time to adopt the vaccination dose, ${ }^{22}$ and hence, triggering of the immune system results in the production of protective antibodies. The subjects enrolling themselves for Covishield vaccination should be aware of these minor manageable side effects. The rescue medicine for the solicited manageable symptoms like fever, pain at the injection site and malaise was advised to take paracetamol, and it was even used as prophylaxis to avoid developing the postvaccination symptoms and hence to increase the acceptance of the COVID-19 vaccine.

\section{Conclusion}

Covishield vaccination in subjects is associated with only minor AEs, and mostly they are manageable with simple measures like the use of over-the-counter drugs like paracetamol. The results of the study are encouraging and can help to decrease the vaccination psychological fear and hence improving compliance to SARS-CoV-2 vaccination. Thus, more participation in mass vaccination program can help to counter COVID-19 pandemic disease by increasing the success rate of vaccination.

\section{Clinical Significance}

It was observed in a noninterventional study that the incidence of solicited and unsolicited AEs was low in our study, and thus, the safety of Covishield vaccine was well established in our study. Thus, the findings observed in our study can be extrapolated for establishing the safety of Covishield in the general population, and so, mass vaccination with Covishield is recommended for the prevention of severe infection with high mortality due to COVID-19 infection.

\section{References}

1. CDC. 2019 novel coronavirus. Wuhan, China: CDC; 2020. Available at: https://www.cdc.gov/coronavirus/2019-ncov/about/index.html [Accessed: January 27, 2020].

2. WHO Director-General's remarks at the media briefing on 2019-nCoV on 11 February 2020. 2020. Available at: https://www.who.int/dg/speeches/ detail/who-director-general-s-remarks-at-the-media-briefing-on-2019ncov-on-11-february-2020 [Accessed: February 13, 2020].

3. CDC. 2019 novel coronavirus, Wuhan, China: symptoms. CDC; 2020. Available at: https://www.cdc.gov/coronavirus/2019-ncov/about/ symptoms.html [Accessed: January 27, 2020]. 
4. WHO coronavirus disease (COVID-19) dashboard. World Health Organization; 2021. Available at: https://covid19.who.int/ [Accessed: February 8, 2021].

5. Kaplan EH. Containing 2019-nCoV (Wuhan) coronavirus. Health Care Manag Sci 2020;23:311-314. DOI: 10.1007/s10729-020-09504-6.

6. India continues saving lives and livelihoods - recording more COVID-19 recoveries while lifting restrictions. Available at: https:// www.who.int/india/emergencies/coronavirus-disease-(covid-19) [Accessed: February 18, 2021].

7. World's largest vaccination programme begins in India on January 16 . The Hindu. January 15, 2021 [Retrieved: January 16, 2021].

8. Moore JP, Klasse PJ. SARS-CoV-2 vaccines: "Warp Speed" needs mind melds not warped minds. J Virol 2020;94(17):1-32. DOI: 10.1128/ JVI.01083-20.

9. Du L, He Y, Zhou Y, et al. The spike protein of SARS-CoV--a target for vaccine and therapeutic development. Nat Rev Microbiol 2009;7(3):226-236. DOI: 10.1038/nrmicro2090.

10. Nath S. Updates on COVID-19 vaccine development and India's roles in vaccine preparation. Int J Sci Res 2020;9(8);137-140.

11. Folegatti PM, Ewer KJ, Aley PK, et al. Safety and immunogenicity of the ChAdOx1 nCoV-19 vaccine against SARS-CoV-2: a preliminary report of a phase $1 / 2$, single-blind, randomised controlled trial. Lancet 2020;396(10249):467-478. DOI: 10.1016/S0140-6736(20)31604-4.

12. Coronavirus disease (COVID-19): vaccines serum - protection from birth onwards. ChAdOx1 nCoV-19 corona virus vaccine (Recombinant) COVISHIELD ${ }^{\mathrm{TM}}$. Available at: https://www.seruminstitute.com/ product_covishield.php.

13. Zhu FC, Li YH, Guan XH, et al. Safety, tolerability, and immunogenicity of a recombinant adenovirus type- 5 vectored COVID-19 vaccine: a dose-escalation, open-label, non-randomized, first-in-human trial. Lancet 2020;395(10240):1845-1854. DOI: 10.1016/S01406736(20)31208-3.

14. Moderna announces positive interim phase 1 data for its mRNA vaccine (mRNA-1273) against novel coronavirus. May 18, 2020. Available at: investors.modernatx.com, https://investors.modernatx. $\mathrm{com} /$ news-releases/news-release-details/moderna-announces- positive-interim-phase-1-data-its-mrna-vaccine authorization by DCGI-CDSCO, MoH and FW on January 3, 2021.

15. DCGI approves Covishield and Covaxin for restricted emergency use in India. DCGI's decision comes after the Subject Expert Committee of CDSCO met on January 1-2, 2021 and recommended the grant of permission for restricted emergency use of these vaccines. Available at: https://www.expresspharma.in/covid19-updates/ dcgi-approves-covishield-and-covaxin-for-restricted-emergencyuse-in-india/.

16. Indian vaccine market report and forecast 2020-2025. Available at: www.imarcgroup.com, https://www.imarcgroup.com/indianvaccinemarket.

17. Knolla MD, Wonodia C. Oxford-AstraZeneca COVID-19 vaccine efficacy. Lancet 2021;397(10269):72-74. DOI: 10.1016/S01406736(20)32623-4.

18. Council for International Organizations of Medical Sciences (CIOMS). Definition and application of terms of vaccine pharmacovigilance (report of CIOMS/WHOWorking Group on VaccinePharmacovigilance). Genève: CIOMS; 2012.

19. Fact sheet for vaccine recipient approved for restricted use in emergency situation of Chadox 1 ncov-19 corona virus vaccine (recombinant). Available at: https://www.seruminstitute.com/pdf/ covishield_fact_sheet.pdf.

20. Voysey M, Costa Clemens SA, Madhi SA, et al. Safety and efficacy of the ChAdOx1 nCoV-19 vaccine (AZD1222) against SARS-CoV-2: an interim analysis of four randomised controlled trials in Brazil, South Africa, and the UK. Oxford COVID Vaccine Trial Group. Lancet 2021;397(10269):99-111. DOI: 10.1016/S0140-6736(20)32661-1.

21. Tapia MD, Sow SO, Mbaye KD. Safety, reactogenicity, and immunogenicity of a chimpanzee adenovirus vectored Ebola vaccine in children in Africa: a randomised, observer-blind, placebocontrolled, phase 2 trial. Lancet Infect Dis 2020;20(6):719-730. DOI: 10.1016/S1473-3099(20)30019-0.

22. Sah R, Shrestha S, Mehta R, et al. AZD1222 (Covishield) vaccination for COVID-19: experiences, challenges, and solutions in Nepal. Travel Med Infect Dis 2021;40:101989. DOI: 10.1016/j.tmaid.2021.101989. 